responses, and research is still far from developing cost-effective and feasible approaches to predict these responses.

Methods Using peripheral blood samples and colonic biopsies, we aim to stratify CD patients into different immunopathotypes. We quantified frequencies of leukocyte populations and cytokines in peripheral blood of patients and healthy controls, using flow cytometry analysis and ELISAs. Cytokine and leukocyte population levels were correlated using Sparse PLS Discriminant Analysis. Additionally, gene expression data was generated by RNAseq from colonic biopsies to identify genes that were differentially expressed between active and remission CD samples, and healthy controls.

Results Our initial analyses have identified 3 distinct clusters of $\mathrm{CD}$ patients based on expression patterns of peripheral blood mononuclear cells (PBMCs) and cytokines. Patient clusters were found to either upregulate pro-inflammatory cytokine only (Cluster A), upregulate pro-inflammatory cytokines with altered frequencies of leukocyte populations (Cluster C) or solely display altered leukocyte frequencies (Cluster C). In order to further understand potential distinct disease mechanisms, significantly differentially expressed genes from the RNAseq data were analysed identifying genes that varied in expression in the CD cohort, resulting in 13 targets. These gene targets are currently validated in additional CD colonic biopsies using qPCR. Together with clinical information about treatment response and disease severity, these data will be used to investigate the capacity of the blood phenotyping and biopsy gene expression signatures to act as biomarkers to predict treatment responses in CD.

Conclusions Based on our results it can be suggested that CD patients have different immunopathotypes, which might be associated with distinct treatment responses. Our ongoing work will assess these potential associations. Should strong associations be identified, their prognostic value will be assessed. Our eventual aim is to help ensure that CD patients receives the most appropriate treatment soon after diagnosis. This is likely to be crucial in limiting damage caused by ongoing inflammation.

\section{PTH-076 SWITCHING FROM ORIGINATOR INFLIXIMAB TO CT-P13: A UK SINGLE CENTRE EXPERIENCE}

${ }^{1}$ Anirudh Pramod Bhandare*, 'Benjamin Crooks, 'Gaurav Bhaskar Nigam, 1,2 Jimmy K Limdi. 'Northern Care Alliance, Manchester, UK; ${ }^{2}$ University of Manchester, Manchester, UK

\subsection{6/gutjnl-2019-BSGAbstracts. 135}

Introduction and Aims The infliximab biosimilar (CT-P13) received market authorization for inflammatory bowel disease in late 2016 with the aim of reducing cost and increasing access to therapy. The prospect of 'switching' patients from originator to CT-P13 has concerned clinicians. We present an experience of 'switching' from originator infliximab (IFX-O) to CT-P13 and present efficacy, safety and immunogenicity data from our cohort.

Methods We performed a retrospective review of patients switched from IFX-O to CT-P13 at our center. Disease demographics, clinical course and outcomes were analysed from electronic case records at 8 months and at last follow-up at 13 months.

Results Ninety-six patients (35 female) were 'switched' from IFX-O to CT-P13. Of these 44 had Ulcerative colitis (UC) and 52 had Crohn's disease (CD) with a mean age at diagnosis of

34.7 years $($ median $=33, \mathrm{IQR}=24.5)$. Montreal phenotype for UC was Proctitis( E1) = 1, Left sided(E2) = 16,Pancolitis $(\mathrm{E} 3)=27$ and for CD $(\mathrm{L} 1=10, \mathrm{~L} 2=12, \mathrm{~L} 3=29$, $\mathrm{L} 4=1)$ and $(\mathrm{B} 1=27, \mathrm{~B} 2=14, \mathrm{~B} 3=11), 9$ patients had perianal disease.

Mean duration of IFX-O treatment before switching was 49.8 months (median $=44, \mathrm{IQR}=52$ ) and on CT-P13 11.5 months (median 13). At switch, 76 patients had a normal $\mathrm{CRP}$ (UC $=33, \mathrm{CD}=43$ ), and in 15 patients it was elevated $(\mathrm{UC}=10, \mathrm{CD}=5)$.

At 8 months, 72 patients $(\mathrm{UC}=34, \mathrm{CD}=38$ ) were in clinical remission (pMayo $<2$ and $\mathrm{HBI}<5$ ) and 80 patients remained in biochemical remission $(\mathrm{UC}=35, \mathrm{CD}=45)$. In 14 patients ( $\mathrm{UC}=8, \mathrm{CD}=6$ ) $\mathrm{CRP}$ increased. Of 51 patients $(\mathrm{UC}=21, \mathrm{CD}=30)$ undergoing endoscopic assessment, 31 achieved mucosal healing ( $\mathrm{UC}=13, \mathrm{CD}=18$ ).

At 13 months 69 patients remained on CT-P13. 28\% discontinued the drug due to immunogenicity $(n=10)$, loss of response $(n=5)$, surgery $(n=5)$, remission $(n=5)$, side effects $(n=2)$ and 1 patient died of hospital acquired pneumonia.

39 out of 96 patients had therapeutic drug levels checked within a median of 13 months from switch. 27 had sub-therapeutic levels (below $4 \mathrm{ug} / \mathrm{ml}$ ),11 of which were switched to another biologic, 5 referred for surgery, 4 had dose escalated to $10 \mathrm{mg} / \mathrm{kg}, 5$ continued CT-P13( 4 with no antibodies seen and 1 with antibodies of 127),one had immunomodulator added and another stopped CT-P13 being in remission. Antibodies to Infliximab were seen in 15 of 39 patients $(38.5 \%)$, of whom 8 were switched to an alternative biologic, 2 had dose escalation (10 mg/kg IFX),4 patients stopped IFX with no other intervention and 1 person continued treatment with low antibody titre of 6 .

Discussion Biosimilar IFX (CT-P13) was well tolerated. Clinical efficacy and loss of response rates with CT-P13 appears to be similar to IFX-O. This holds promise for a wider adoption of 'switching' to fulfil the purported aims of wider access to treatment at a lower cost.

\section{PTH-077 DE-ESCALATING THERAPY IN PATIENTS WITH CROHN'S DISEASE RECEIVING ADALIMUMAB: SUBGROUP ANALYSIS OF THE CALM STUDY}

${ }^{1} \mathrm{P}$ Bossuyt ${ }^{*},{ }^{2} \mathrm{G} \mathrm{D}^{\prime}$ Haens, ${ }^{3} \mathrm{R}$ Panaccione, ${ }^{4} \mathrm{~W}$ Sandborn, ${ }^{5} \mathrm{~W}$ Reinisch, ${ }^{6} \mathrm{~S}$ Travis, ${ }^{7} \mathrm{~S} S$ chreiber, ${ }^{8} \mathrm{~A}$ Armuzzi, ${ }^{9} \mathrm{~S}$ Danese, ${ }^{10} \mathrm{~S}$ Berg, ${ }^{11} \mathrm{~J}$ Petersson, ${ }^{11} \mathrm{~J}$-F Maa, ${ }^{11} \mathrm{E}$ Neimark, ${ }^{12} \mathrm{~J}$ F Colombel. ' Imelda General Hospital, Bonheiden, Belgium; ${ }^{2}$ Academic Medical Center, Amsterdam, Netherlands; ${ }^{3}$ University of Calgary, Calgary, Canada; ${ }^{4}$ University of California San Diego, La Jolla, US; ${ }^{5}$ Medical University of Vienna, Vienna, Austria; ${ }^{6}$ Oxford University Hospitals, Oxford, UK; ${ }^{7}$ University Hospital Schleswig-Holstein, Kiel, Germany; ${ }^{8}$ Fondazione Policlinico Gemelli Università, Rome, Italy; ${ }^{9}$ Humanitas University, Rozzano, Italy; ${ }^{10} \mathrm{AbbVie}$, Solna, Sweden; ${ }^{11}$ AbbVie Inc, North Chicago, US; ${ }^{12}$ Icahn School of Medicine at Mount Sinai, New York, US

\subsection{6/gutjnl-2019-BSGAbstracts. 136}

Introduction This analysis evaluated the impact of de-escalating therapy on mucosal healing 48 weeks (wks) after randomization in patients (pts) with Crohn's disease (CD) in the CALM study.

Methods Pts with moderate-to-severe CD naïve to immunomodulators and biologics were randomized 1:1 to a tight control group (TCG) or clinical management group (CMG) after 8 wks of prednisone therapy. Treatment was escalated from no 